\title{
Réalisation d'un diagnostic territorial de développement durable. Expérimentation dans le Bassin potassique alsacien
}

\section{François Besancenot}

\section{(2) OpenEdition}

Journals

\section{Édition électronique}

URL : http://journals.openedition.org/developpementdurable/6083

DOI : 10.4000/developpementdurable.6083

ISSN : 1772-9971

Éditeur

Association DD\&T

\section{Référence électronique}

François Besancenot, « Réalisation d'un diagnostic territorial de développement durable.

Expérimentation dans le Bassin potassique alsacien », Développement durable et territoires [En ligne], Varia (2004-2010), mis en ligne le 12 juin 2008, consulté le 19 avril 2019. URL : http://

journals.openedition.org/developpementdurable/6083; DOI : 10.4000/developpementdurable.6083

Ce document a été généré automatiquement le 19 avril 2019.

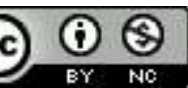

Développement Durable et Territoires est mis à disposition selon les termes de la licence Creative Commons Attribution - Pas d'Utilisation Commerciale 4.0 International. 
Réalisation d'un diagnostic territorial de développement durable. Expérimentation dans le Bassin potassique alsacien

\author{
François Besancenot
}

1 Le Bassin potassique alsacien, territoire minier du sud de la plaine d'Alsace, a fait le choix dès sa reconversion en 1994 d'un nouveau modèle de développement (figure 1). 
Fig. 1: Situation du Bassin potassique alsacien

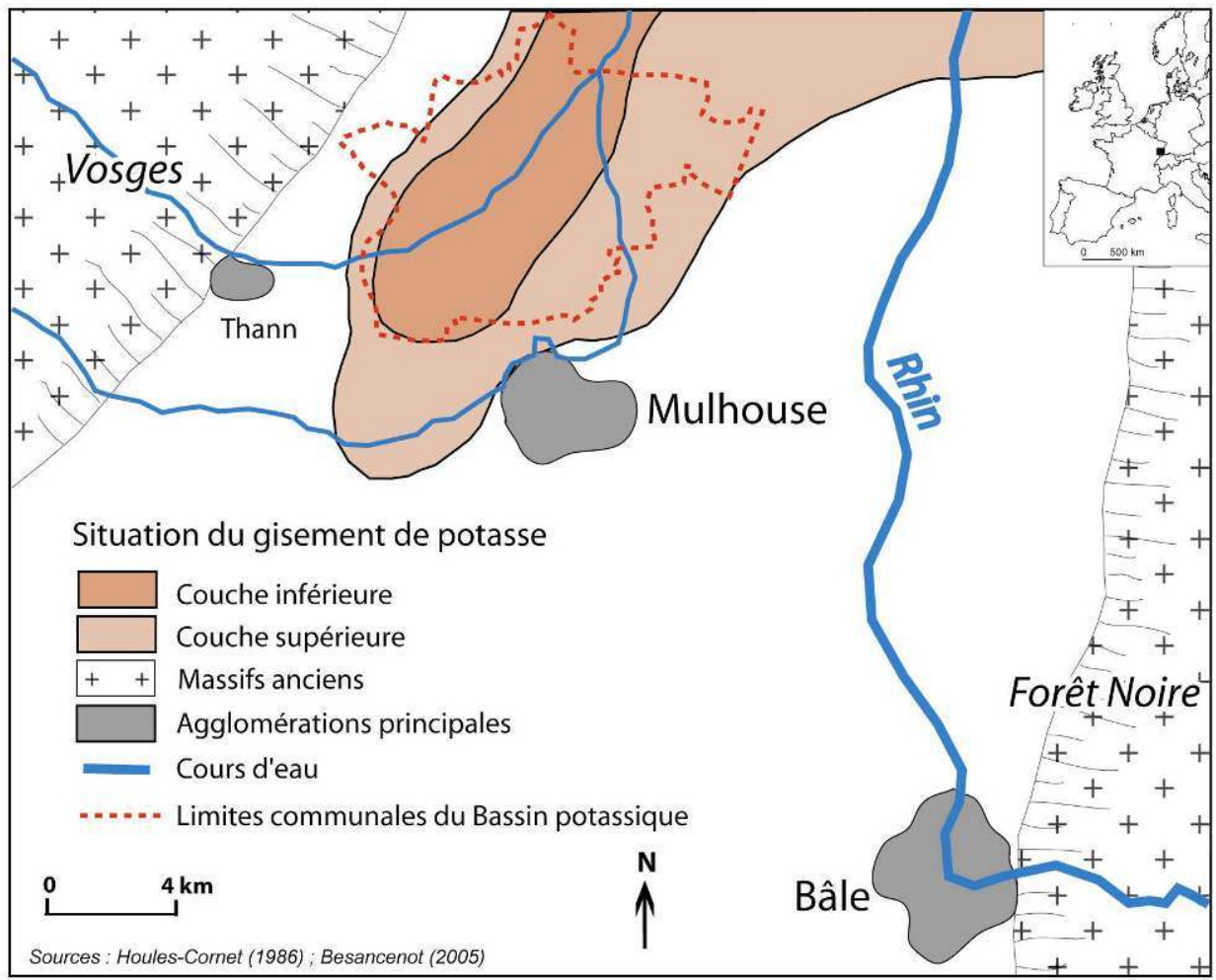

Les acteurs politiques ainsi que les responsables des Mines de Potasse d'Alsace (MDPA) ont alors décidé une autre dynamique de développement pour le bassin. Elle ne sera plus désormais fondée sur une seule ressource naturelle épuisable, la potasse, mais sur des facteurs de développement plus durables. La question est de savoir comment envisager la réussite d'un tel passage. Ce problème ne concerne pas seulement le Bassin potassique mais aussi bon nombre de collectivités territoriales qui, malgré la mise à disposition de nombreux outils tel l'Agenda 21 local, se posent encore la question de la mise en œuvre du développement durable sur leur territoire.

Réfléchir sur une démarche pertinente de développement territorial durable est donc une priorité. En faisant du Bassin potassique notre territoire test, l'objectif est d'adapter à tout type de territoire une même démarche de diagnostic de développement durable. Il s'agit dans un premier temps d'expliciter le contenu du diagnostic, de justifier le choix du Bassin potassique comme territoire pertinent d'étude et de projet puis de confronter la théorie aux réalités du terrain. Un bilan mettant en avant les difficultés et les facilités de ce type de démarche pourra alors être proposé.

Inspiré de la démarche du Centre d'Etude sur les Réseaux, les Transports et l'Urbanisme (CERTU), le diagnostic territorial de développement durable est " une démarche stratégique de développement d'un territoire (...) préparant le débat concernant un espace habité et doué d'une identité, aménagé et supposant des logiques de développement dans une perspective de développement durable» (Rouxel, 2000). Il précède les stratégies et le programme d'action de l'Agenda 21 local. Il se doit de respecter cinq étapes essentielles: le choix d'un territoire pertinent d'étude et de projet, la définition des objectifs de développement territorial durable, le choix des spécificités du territoire, la confrontation des spécificités aux objectifs permettant d'identifier les problématiques et les indicateurs de 
développement durable (IDD) qui leur correspondent et enfin la désagrégation de ces derniers permettant leur évaluation (Figure 2).

Figure 2: Les étapes du diagnostic territorial de développement durable

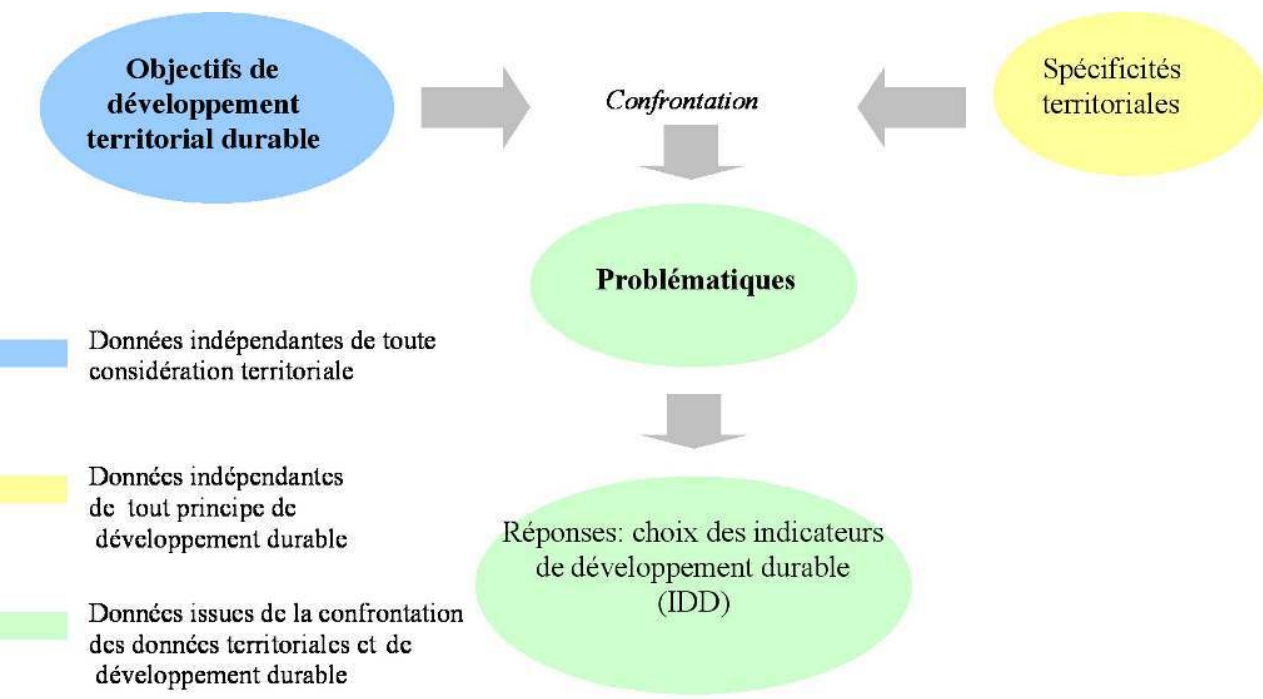

D'après ROUXEL, 2000 et MEDD, 2002

5 soit théoriquement optimal dans la perspective d'un plan satisfaisant de développement durable ? S'il est vain de chercher un territoire dans lequel les différentes échelles de temps, d'espace, de domaines (social, environnemental et économique) et de décisions s'inscrivent au sein d'un même système, quelle serait dans ce cas la solution la moins dommageable pour la durabilité ? Le problème est d'autant plus difficile à résoudre que le développement durable ne peut se réaliser pleinement dès l'instant qu'il n'est pas globalisé. Les territoires, naturellement limités géographiquement, ne peuvent traiter de tous les enjeux du concept à eux seuls. Le développement durable territorialisé diffère donc d'un développement durable globalisé sur trois aspects essentiels: les interdépendances entre territoires, la spécialisation des territoires et la territorialisation des autorités de tutelle (Nijkamp, Lasschuit et Soeteman, 1992). Ainsi, appliquer les principes globaux de la durabilité à l'échelle d'une commune ou d'un canton relève de l'utopie. A plus petite échelle en revanche, le développement durable peut se réaliser plus aisément dans la mesure où un territoire contenu peut être sacrifié pour assurer la durabilité du territoire contenant (Nijkamp, Lasschuit et Soeteman, 1992): la prise en compte de deux territoires $\mathrm{A}$ et $\mathrm{B}$, l'un bénéficiaire (sur un plan énergétique par exemple), l'autre déficitaire par rapport à $\mathrm{A}$, risque d'être biaisée si l'on ne prend pas en compte un autre territoire $C$ permettant de compenser le déficit de B (Zuindeau, 2000). Là est toute la complexité des externalités (effets économiques externes positifs ou négatifs).

6 Si l'on cherche à mener à bien un projet de développement durable "idéal" même à l'échelon d'une collectivité territoriale, le monde global nous contraint, par l'éloignement considérable des prélèvements et des apports qu'il suppose, à une analyse territoriale et systémique des plus complexes. Ces problèmes soulèvent d'ailleurs chez Jacques Theys (2001), dans son étude sur les indicateurs, plusieurs questions: "[...] par quel système de mesure représenter les relations entre stock et flux de ressources : évaluations économiques, bilan 
matières/énergie, cycle de renouvellement? Y a-t-il des moyens simples pour évaluer les "échanges extérieurs" de ressources et de pollutions?" Dans ce cas, il est légitime de se demander si, dans le cadre d'un diagnostic de développement durable, il est nécessaire de prendre en compte des externalités aussi lointaines, sachant que le niveau de complexité de ce calcul est si élevé qu'il risque d'occulter un certain nombre de réalités individuelles et collectives. Bertrand Zuindeau (1997) minimise l'écueil du choix d'un territoire idéal. Par la quasi impossibilité de "compenser une surexploitation de ressources en un territoire donné par un flux positif de cette même ressource en un autre territoire ", la question qui est ici posée "est celle de "l'espace minimal de durabilité" autorisant des compensations internes à l'espace en question."

7 A chacune des problématiques de développement durable devra donc correspondre un territoire d'étude donné, géré par une autorité de tutelle clairement identifiée. Affirmer la pertinence ou non d'un territoire de projet semble donc cavalier tant qu'il n'y a pas eu de suivi ni d'évaluation des indicateurs de développement durable. Mais parce qu'il faudra bien commencer le diagnostic, il conviendra d'opter pour une approche certes empirique mais néanmoins pertinente. Le développement durable devra donc idéalement s'inscrire dans un territoire auquel la majorité des habitants se sent appartenir. Cet espace devra, dans la mesure du possible, réunir la plupart des paramètres qui font qu'un territoire est cohérent. Le territoire sera donc sélectionné en fonction de sa première dimension constitutive : l'identité. Un espace donné est en effet territoire dès lors qu'un collectif humain se l'approprie et le pense comme tel (Lévy et Lussault, 2003).

Il s'agit de décrire la démarche permettant d'aboutir au choix d'IDD. Chaque étape sera décrite successivement: du choix des objectifs de développement territorial durable à l'élaboration des IDD en passant par l'identification des spécificités du territoire d'étude.

Si le développement durable tend à garantir un modèle de société soutenable, alors l'espace lui est indissociable. Le lien légitime entre territoire et durabilité ainsi que la nécessité pour le développement durable de considérer le territoire dans sa globalité, nous conduisent à une indentification des interdépendances territoriales. Quatre principes de durabilité ont été relevés. Ils aspirent tous à un équilibre entre les échelles d'espaces (un territoire doit prendre en compte dans son propre développement les intérêts des autres), les échelles de temps (l'acteur se doit de considérer conjointement des projets de court, de moyen et de long terme mais aussi se servir de l'expérience du passé pour mieux gérer le présent et préparer l'avenir), les domaines social, environnemental et économique, et, enfin, les acteurs de diverses spécialités, raisonnant sur des échelles d'espaces et de temps différentes. Ces principes sont intrinsèquement territoriaux. Afin de prendre en compte les différentes composantes du territoire, tout en évitant une analyse trop exhaustive et éminemment complexe, les objectifs de développement durable, valables quel que soit le territoire, s'inscrivent dans les cinq sous-systèmes territoriaux -l'habitation, l'appropriation, l'exploitation, la communication et la gestion - identifiés par le géographe Roger Brunet (1990). Dans le Bassin potassique par exemple, l'habitation correspond à la cité minière, l'appropriation au paysage minier, l'exploitation aux aménagements de zones d'activités, la communication aux échanges entre acteurs et "simples" citoyens et la gestion aux actions engagées par l'espace administratif de projet, ici la Communauté d'Agglomération de Mulhouse Sud-Alsace (CAMSA). Un objectif permanent de développement durable s'insèrera donc dans un sous-système territorial donné (Figure 3). 
Figure 3: Des sous-systèmes territoriaux aux objectifs de développement territorial durable

\begin{tabular}{|l|l|}
\hline Sous-systèmes territoriaux & \multicolumn{1}{|c|}{ Objectifs de développement territorial durable } \\
\hline Habitation & $\begin{array}{l}\text { Garantir l'intégrité du lieu d'habitation, à savoir l'intégrité } \\
\text { humaine à travers son corps, son esprit et son habitat privé }\end{array}$ \\
\hline Appropriation & $\begin{array}{l}\text { Préserver et valoriser le patrimoine individuel et collectif } \\
\text { appropriè pratiquement et psychologiquement }\end{array}$ \\
\hline Exploitation & Préserver et valoriser les ressources \\
\hline Echange & Entretenir et améliorer les relations humaines \\
\hline Gestion & Organiser la gestion du territoire \\
\hline
\end{tabular}

Les spécificités territoriales "ressortent d'un état sommaire de connaissance résultant du terrain, d'entretiens avec les principaux acteurs locaux et de lectures des documents existants " (Rouxel, 2000). Cet exposé précède la confrontation des spécificités aux objectifs du développement durable et par conséquent la formulation des problématiques de développement durable propres au Bassin potassique alsacien. Les spécificités sont donc un état des lieux du territoire. Leur énoncé ne doit en aucun cas être exhaustif. Elles doivent in fine orienter les problématiques de développement durable. Celles retenues pour le bassin potassique ont été classées selon cinq grands thèmes correspondant chacun à un sous-système territorial. Ce type de classement doit favoriser la confrontation des spécificités aux objectifs classés selon le même modèle.

11 Les problématiques, issues de la confrontation entre objectifs permanents de développement durable et spécificités du territoire étudié, doivent aboutir à une batterie d'Indicateurs de développement durable (IDD) permettant de trouver des solutions à ces questions. L'IDD n'est pas un indicateur brut, mesurable directement et sans agrégation préalable. C'est un moyen de répondre à une problématique de développement durable territorialisé, " produit ultime du travail de recherche, compte tenu de la référence à l'action que contient la notion de développement durable» (Jollivet, 2001). Parce que les IDD, pistes d'investigation permettant de répondre aux problématiques, ne sont à même d'être évalués ou mesurables, il est nécessaire de compléter la démarche par la désagrégation des IDD en indicateurs mesurables.

12 Les indicateurs bruts, qui interviennent après le choix des IDD, permettront, une fois évalués et agrégés, de mesurer le niveau de durabilité de l'indicateur composite. C'est l'évaluation préalable des indicateurs bruts qui sera à même d'aboutir à une agrégation pertinente (Figure 4). 
Figure 4: Problématiques et désagrégation des IDD

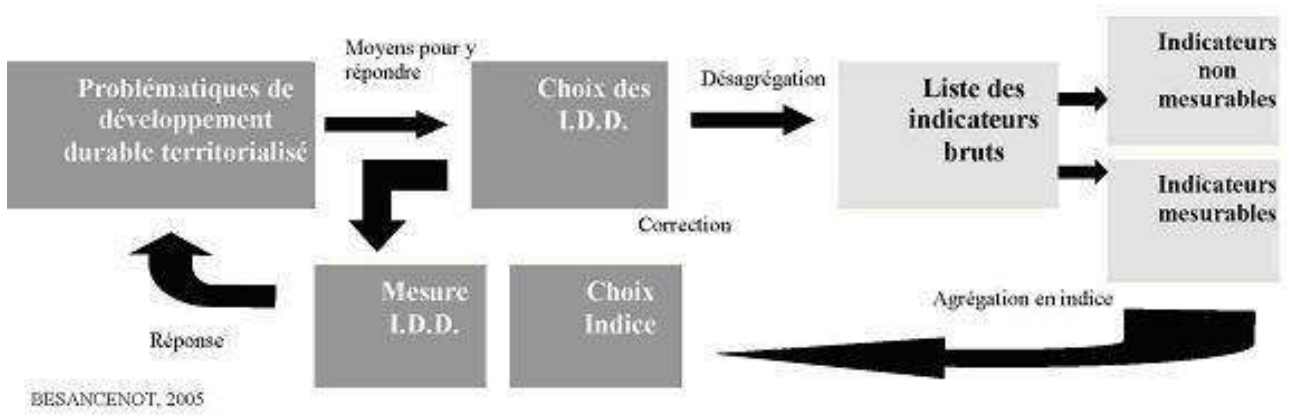

13 Afin d'éviter toute agrégation factuelle, respecter les principes de durabilité, permettre une analyse plus judicieuse et plus opérationnelle pour la collectivité locale ou territoriale et enfin répartir les sous-indicateurs bruts selon les cinq sous-systèmes territoriaux, le modèle DPSIR des Nations Unies (Driving force-Pressure-State-ImpactResponse traduit DPEIR : forces Directrices-Pression-Etat-Impact-Réponse) (Portail de l'Environnement, 2004) a été retenu. Ici, toute la chaîne des effets nécessaires à l'évaluation des politiques est illustrée d'indicateurs. Par exemple, les activités humaines, les secteurs économiques, la consommation, la démographie, les technologies sont des forces directrices du système représenté. Ces activités exercent des pressions. Par conséquent, l'état (des comportements environnementaux par exemple) en est affecté. En aval, ces changements d'état influencent la santé des êtres vivants et des systèmes de ressources. En considérant le profil de ces catégories, et particulièrement celui des impacts, une réponse correctrice de la société est proposée. Ces réponses, qu'elles soient de nature réglementaire, économique ou volontaire, influencent à leur tour la configuration du système (Zaccaï, 2004). A chaque IDD correspond donc l'agrégation de plusieurs indicateurs bruts répartis selon les cinq intitulés: force Directrice, Pression, Etat, Impact et Réponse. L'évaluation préalable de ces sous-indicateurs DPEIR, selon des normes préétablies, donnera lieu, après agrégation, à une évaluation de l'IDD.

14 Le principe d'interdépendance propre au développement durable devra être respecté. Dans une logique relationnelle entre échelles de temps, les différents sous-indicateurs de tels ou tels objets spatiaux ne correspondent pas forcément au même référentiel temps. Chaque sous-indicateur influence le suivant, ce qui permet de prévoir à court, moyen ou long terme les effets de telle ou telle action sur l'un d'entre eux. De plus, les effets produits sur certains sous-indicateurs, qui relèvent d'actions passées, permettent de comprendre la valeur actuelle des autres et ainsi de prévoir leur évolution. L'interdépendance des échelles d'espace fait que les sous-indicateurs ne se trouvent ni ne se mesurent forcément sur le même espace. L'interdépendance des domaines montre que 
les sous-indicateurs d'une même chaîne d'action DPEIR ne sont pas nécessairement exclusivement sociaux, environnementaux ou économiques. Ce système bouclé met ainsi en relation des sous-indicateurs de différents domaines. Enfin, dans un souci d'interdépendance des échelles décisionnelles, chaque sous-indicateur relèvera de la gestion et du suivi d'un acteur en particulier. Il peut s'agir d'une administration, une entreprise, une association, de nature et de niveaux hiérarchiques différents.

Le diagnostic a fait l'objet d'un test dans le Bassin potassique alsacien. Avant d'exposer les conclusions de ce travail de recherche et donc d'estimer la faisabilité de la démarche, il est nécessaire de montrer que ce territoire a été choisi à juste titre pour l'élaboration d'un plan de développement durable.

Puisqu'un territoire de projet pertinent est d'abord un espace cohérent sur le plan identitaire, c'est l'importance des paramètres d'identification des habitants au Bassin potassique qui a ici retenu notre attention. Il est ainsi fait référence à la capacité qu'a le territoire minier d'accueillir un projet unanime de société, condition nécessaire au choix d'un plan de développement durable pertinent. Une évaluation de sa cohésion identitaire a donc été opérée. Afin de déterminer lequel, des facteurs territoriaux de cohésion et d'émiettement, a eu le plus de retentissement, trois enquêtes ont été menées. La première a eu lieu en 2001 sur un échantillon représentatif de 993 personnes. La seconde s'est déroulée en 2004 et a concerné un échantillon de 33 personnes réparties selon plusieurs critères : l'âge, la catégorie socio-professionnelle, le niveau d'investissement dans la vie publique et enfin le niveau de responsabilité territoriale. La troisième, réalisée en juillet 2005, n'a concerné que les maires des douze communes minières. La seconde comme la troisième a permis d'objectiver certaines perceptions et représentations de 2001.

Les conclusions des enquêtes ont mis en évidence la volonté d'une grande majorité des habitants de conserver des traces de la mine, de léguer à la Communauté de Communes du Bassin Potassique (CCBP) la gestion de la reconversion et par là même la prise en charge du territoire minier et enfin de fédérer les douze communes minières au sein d'un même territoire de projet. L'enquête de 2001 a montré que la plupart des habitants se sentaient prioritairement appartenir à l'entité Bassin potassique, bien avant leur village et leur cité minière.

18 La superposition presque parfaite, jusqu'à la disparition de la CCBP en 2004, de l'espace identitaire et de l'espace de projet, a aussi permis d'affirmer la cohérence administrative du territoire. Même si certaines communes, émancipées de la mine depuis fort longtemps ou encore essentiellement polarisées par l'agglomération mulhousienne, trahissent un attachement moindre au Bassin potassique et à son maintien en tant que territoire de projet.

19 Malgré l'intégration de dix communes du Bassin potassique à la CAMSA en 2004, la consultation des habitants entre 2001 et 2005 a néanmoins prouvé que le territoire minier était un véritable espace identitaire, d'autant plus visible que cet espace laisse entrevoir sa dimension territoriale profonde, «dimension cachée (...) qui peut resurgir en période de crise économique et sociale» (Sauvin, 1998). Le bassin a donc pu être considéré comme un territoire d'étude pertinent à prendre en compte dans la mise en œuvre par la CAMSA d'un diagnostic territorial de développement durable.

20 Les spécificités, tout d'abord, ont été identifiées à partir des perceptions et des représentations des habitants recueillies lors des enquêtes de 2001, 2004 et 2005 mais aussi par les données provenant de sources plus officielles (rapports publiés par les 
MDPA, la CCBP, la CAMSA et les entretiens effectués auprès de certains responsables politiques). N'ont été retenus que les paramètres à tendance lourde, c'est-à-dire ceux dont l'inertie a eu suffisamment d'impact pour s'épargner tout changement brutal dans le temps. Ils se réfèrent à l'identité minière, à la qualité du cadre de vie, à l'histoire du Bassin potassique, etc. Seules les caractéristiques mettant en lumière la singularité du territoire minier ont été abordées.

21 Les problématiques de développement durable, choisies au regard des spécificités du territoire d'une part, et de l'ensemble des objectifs permanents du développement durable d'autre part, ont été validées en 2005 par les maires du Bassin potassique ainsi que par certains responsables de la CAMSA. Une liste de thèmes à aborder, classés selon les objectifs permanents de développement durable, leur a été soumise. Ceux considérés comme secondaires ou inexistants au sein d'enjeux actuels ont été supprimés. D'autres ont été conservés ou ajoutés. Les thématiques -permettant d'occulter aucun champ thématique dans le questionnement mais aussi de classer méthodiquement les IDD - et les IDD ont ensuite été sélectionnés (Figure 5).

Figure 5 : Des objectifs aux IDD: l'exemple des ensembles architecturaux remarquables

\begin{tabular}{|c|c|c|c|}
\hline Objectif & Problématique & Thématique & IDD \\
\hline \multirow{3}{*}{$\begin{array}{l}\text { Préserver et } \\
\text { valoriser le } \\
\text { patrimoine individuel } \\
\text { et collectif approprié } \\
\text { pratiquement et } \\
\text { psychologiquement }\end{array}$} & \multirow{2}{*}{$\begin{array}{l}\text { Comment préserver et } \\
\text { améliorer le paysage } \\
\text { architectural inféodé à la mine } \\
\text { ? Comment préserver les } \\
\text { références symboliques liées } \\
\text { à la mine? }\end{array}$} & $\begin{array}{l}\text { Qualité des } \\
\text { paysages }\end{array}$ & $\begin{array}{l}\text { Qualité et pérennité des paysages } \\
\text { industriels et miniers: terrils, cités } \\
\text { minières, bâtiments miniers } \\
\text { remarquables, Ecomusée/ } \\
\text { développement du souvenir de la } \\
\text { mine }\end{array}$ \\
\hline & & $\begin{array}{l}\text { Volonté de } \\
\text { préservation }\end{array}$ & $\begin{array}{l}\text { Souhait pour la population de } \\
\text { conserver des traces de l'activité } \\
\text { minière }\end{array}$ \\
\hline & $\begin{array}{c}\text { Comment préserver et } \\
\text { améliorer le paysage pré- } \\
\text { minier? Comment préserver } \\
\text { ces références symboliques? }\end{array}$ & $\begin{array}{l}\text { Qualité des } \\
\text { paysages }\end{array}$ & $\begin{array}{l}\text { Qualité et pérennité des ensembles } \\
\text { architecturaux pré-miniers }\end{array}$ \\
\hline
\end{tabular}

Les enjeux les plus fréquemment évoqués dans les problématiques sont: la préservation d'une certaine qualité de vie propre au bassin, le maintien des intérêts de ce territoire ainsi que le maintien et le développement de son activité complété par la mise en place d'une certaine spécificité de développement. Précisons que l'enjeu de la coopération intercommunale pour une reconversion réussie revêt une importance capitale aux yeux des habitants du bassin. C'est aussi parce que la reconversion a profondément et durablement marqué les esprits : les enjeux les plus urgents et les plus cruciaux ont davantage concerné l'emploi et son développement, la préservation d'une certaine qualité de vie inhérente au paternalisme MDPA et la volonté de reconvertir, dans les meilleures conditions, le Bassin potassique via les aides de la Région Alsace, de l'Etat et de l'Union-Européenne. S'ensuit un enjeu tout aussi important: la capacité des communes minières à se fédérer, afin d'organiser au mieux la reconversion. D'autres enjeux semblent moins urgents, tels la préservation du patrimoine minier, le maintien d'une certaine sociabilité propre au Bassin potassique, le maintien et la promotion du souvenir lié au passé minier voire pré-minier, la question des transports collectifs, la conservation d'une certaine mixité sociale, la découverte et la connaissance de son territoire, la préservation $\mathrm{du}$ patrimoine propre aux MDPA et enfin la sensibilisation au développement durable et à la cohésion territoriale. 

indicateur de réponse " $\mathrm{R}$ " n'apparaît pas encore dans la démarche étant donné que l'évaluation n'a pas encore eu lieu) et de les soumettre aux élus de la CAMSA et des différentes communes du Bassin potassique. Les sous-indicateurs ont été sélectionnés à partir d'indicateurs bruts mesurables produits par plusieurs organismes internationaux (Organisation de Coopération et de Développement Economique, Organisation des Nations Unies, etc.), d'Etat (Agence de l'environnement et de la maîtrise de l'énergie, office Nationale des Forêts, Institut National de la Statistique et des Etudes Economiques, etc.) et régionaux (Direction régionale de l'environnement, Direction Régionale de l'Industrie, de la Recherche et de l'Environnement, etc.). Ce sont les maires des douze communes du Bassin potassique ainsi que les personnes en charge de l'Agenda 21 local CAMSA qui ont fait part de leurs critiques et apporté des rectifications à la liste d'IDD et de sous-indicateurs. DPEI. L'IDD serait représenté par un sous-indicateur d'Etat: «classement du bâtiment», subissant les pressions mesurées par un sous-indicateur de Pression : «valeur foncière des terrains", lui-même engendré par une force positive ou force Directrice: "demande foncière ». L'indicateur d'Etat provoque des Impacts : «plaintes » exprimées notamment par les personnes attachées au patrimoine minier, plaintes elles-mêmes relativisées par un indicateur légitimant la pression, la force Directrice (Figure 6).

Figure 6 : Désagrégation de l'IDD «Qualité et pérennité des paysages industriels et miniers et des ensembles architecturaux pré-miniers »)

\begin{tabular}{|c|c|c|c|c|c|c|c|c|}
\hline IDD & $\begin{array}{c}\text { Force } \\
\text { Directice }\end{array}$ & Lien D-P & Pression & Lien P-E & Etat & Lien E-I & Impact & Lien I-D \\
\hline $\begin{array}{l}\text { Qualité et } \\
\text { pérennité des } \\
\text { paysages } \\
\text { industriels et } \\
\text { miniers et des } \\
\text { ensembles } \\
\text { architecturaux } \\
\text { pré-miniers }\end{array}$ & $\begin{array}{l}\text { Besoins } \\
\text { fonciers et } \\
\text { immobiliers }\end{array}$ & $\begin{array}{l}\text { Légitimité } \\
\text { de la } \\
\text { pression } \\
\text { foncière au } \\
\text { regard des } \\
\text { besoins } \\
\text { fonciers, } \\
\text { immobiliers }\end{array}$ & $\begin{array}{l}\text { Pression } \\
\text { foncière : } \\
\text { valeurs } \\
\text { foncières }\end{array}$ & $\begin{array}{l}\text { Relations de } \\
\text { cause à effet } \\
\text { entre } \\
\text { pression } \\
\text { foncière et } \\
\text { qualité et } \\
\text { pérennitè des } \\
\text { paysages } \\
\text { industriels et } \\
\text { miniers et des } \\
\text { ensembles } \\
\text { architecturaux } \\
\text { pré-miniers }\end{array}$ & $\begin{array}{l}\text { Qualité et } \\
\text { pérennité des } \\
\text { paysages } \\
\text { industriels et } \\
\text { miniers: } \\
\text { terrils, cités } \\
\text { minières, } \\
\text { bâtiments } \\
\text { miniers } \\
\text { remarquables, } \\
\text { Ecomusée } \\
\text { qualité de } \\
\text { pérennité des } \\
\text { ensembles } \\
\text { architecturaux } \\
\text { pré-miniers }\end{array}$ & $\begin{array}{l}\text { Relations de } \\
\text { cause à effet } \\
\text { entre qualité } \\
\text { et pérennité } \\
\text { des paysages } \\
\text { industriels et } \\
\text { miniers et des } \\
\text { ensembles } \\
\text { architecturaux } \\
\text { pré-miniers et } \\
\text { préférences, } \\
\text { insatisfactions } \\
\text { déclarées }\end{array}$ & $\begin{array}{l}\text { Préférences } \\
\text { et } \\
\text { insatisfactions } \\
\text { déclarées }\end{array}$ & $\begin{array}{l}\text { Légitimité } \\
\text { des } \\
\text { observations, } \\
\text { plaintes au } \\
\text { regard des } \\
\text { besoins } \\
\text { fonciers et } \\
\text { immobiliers }\end{array}$ \\
\hline
\end{tabular}

Les sous-indicateurs existants mais non opérationnels, sélectionnés par la CAMSA, ont tout de même été signalés. Afin de réfléchir sur la manière de mettre en relation les sousindicateurs, situés les uns à côté des autres dans une chaine d'effets DPEI, des intitulés ont été donnés aux liens D-P, $P-E, E-I$ et I-D. Rappelons que la chaîne d'effets DPEI, pour être conforme au principe d'interdépendance, doit être bouclée. C'est pourquoi le lien I-D est aussi mentionné. Enfin, un grand nombre de sous-indicateurs n'est pas présent dans le tableau. La raison est que les indicateurs bruts correspondants n'existent pas. D'ailleurs, lorsque pour chaque IDD aucun sous-indicateur n'existe, l'IDD n'est pas signalé.

Le foisonnement d'indicateurs bruts disponibles n'a pas pour autant rendu possible le remplissage de toutes les cases laissées vacantes par des sous-indicateurs qui ne sont pas assez pertinents pour la chaîne d'effets DPEI. Les liens qui unissent les sous-indicateurs entre eux ne relèvent pour l'instant que de considérations théoriques. Les producteurs de deux sous-indicateurs situés l'un à côté de l'autre dans une logique DPEI, entretiennent 
d'ailleurs très rarement de relations entre eux! Dès lors, ils ne peuvent encore moins mettre en relation leurs sous-indicateurs respectifs. A cela s'ajoute bien entendu la difficulté à reconstituer pour certains IDD une boucle DPEI plus que pour d'autres.

La démarche diagnostique telle qu'elle est présentée ici est envisageable pour le Bassin potassique mais aussi pour tout autre territoire cohérent sur le plan identitaire et prêt à s'investir dans un plan de développement durable, via l'Agenda 21 local.

Il est d'abord nécessaire de respecter chaque étape du diagnostic, du choix des spécificités à l'inventaire des IDD. Il convient ensuite de l'intégrer à l'Agenda 21 local, en amont des stratégies et des actions décidées par l'espace de projet. On a constaté que le développement durable dans le Bassin potassique était alors possible et que sa territorialité pouvait être préservée et valorisée quel que soit son espace de décision. Cela veut dire que dans le cadre d'un diagnostic, le choix d'un territoire d'étude est nécessaire mais pas suffisant. De plus, certains progrès restent à faire quant au choix du comité de pilotage en charge de l'Agenda 21 local et donc du diagnostic et quant à la concertation et la coordination permettant de sélectionner les objectifs, les spécificités territoriales, les problématiques et enfin les IDD.

Si toutes les étapes du diagnostic sont aujourd'hui envisageables quel que soit le territoire étudié, l'évaluation semble faire défaut. La tentative de mise en œuvre du diagnostic dans le Bassin potassique a trahi l'impossibilité d'envisager une évaluation des IDD par le biais de leurs sous-indicateurs DPEI. Ces derniers ne sont en grande majorité ni disponibles, ni mesurables, ni capables pour le moment d'être mis en relation dans une logique DPEI; les administrations demandeuses étant le plus souvent insuffisamment préparées à leur utilisation. Toutefois, plusieurs idées émanent de cette étude. Il serait souhaitable non seulement de poursuivre les recherches dans le domaine de l'efficacité évaluative mais aussi dans une communication dédiée à l'aspect fonctionnel de la démarche permettant d'aboutir à des IDD opérationnels.

\section{BIBLIOGRAPHIE}

Besancenot F., 2006, Durabilité, territorialité : un seul enjeu ? Réflexion sur la mise en place d'un développement territorial durable à partir d'un exemple : le Bassin potassique alsacien, Thèse de doctorat en géographie, Ecole Normale Supérieure Lettres et Sciences humaines, Lyon, 535 p + annexes.

Brunet R., 1990, « Le déchiffrement du monde », Mondes nouveaux, in Brunet (dir.), Géographie Universelle, tome 1, Livre premier, Paris, Hachette-Reclus, p. 9-271.

Houles-Cornet A., 1986, Un espace industriel en voie de restructuration: le Bassin potassique d'Alsace, Thèse de doctorat en géographie, ULP, Strasbourg, $330 \mathrm{p}$.

Jollivet M., 2001, Le développement durable, de l'utopie au concept. De nouveaux chantiers pour la recherche, Paris, Elsevier, coll. Environnement, $288 \mathrm{p}$

Lévy J., Lussault M., (dir.), 2003, Dictionnaire de la géographie et de l'espace des sociétés, Saint-Just-la Pendue, Belin, $1034 \mathrm{p}$. 
MEDD (Ministère de l'Ecologie et du Développement Durable), novembre 2002, Des outils et des démarches pour l'élaboration d'un agenda 21 local. Le diagnostic territorial inscrit dans une démarche de développement durable, Suivi de l'Appel à projets les outils et les démarches en vue de la réalisation d'Agenda 21 locaux, $25 \mathrm{p}$.

Miossec A., Arnould P., Veyret Y. (dir. scientifique), 2004, « Vers une géographie du développement durable », Historiens et Géographes, № 387, Union Géographique Internationale, Comité National Français de Géographie, 260 p.

Nijkamp P. , Lasschuit P., Soeteman F., 1992, « Sustainable Development in a regional System », in Breheny Michael. J. (dir.), Sustainable Development and Urban Form, London, Series editor P.W.J. Batey, p. 39-66.

Portail de l'Environnement (2003), Illustration du modèle DPSIR, www.environnement.public.lu/ glossaire/DPSIR/, 1 p.

Rouxel F., Rist D. 2000, Le développement durable, approche méthodologique dans les diagnostics territoriaux, Lyon, Centre d'études sur les réseaux, les transports, l'urbanisme et les constructions publiques, éd. CERTU, $147 \mathrm{p}$.

Sachs I., 2002, « Une civilisation de l'être », in Ducroux A.-M., Les Nouveaux utopistes du développement durable, Paris, Ed. Autrement, Coll. Mutations n² 216, p. 27-39.

Sauvin T., mars 1998, « L'assise territoriale du développement durable », Natures-Sciences-Sociétés, vol. $6 \mathrm{n}^{\circ} 2$, Paris, Elsevier, p. 55-58.

Theys J., 2001, « A la recherche du développement durable : un détour par les indicateurs », in Jollivet M., « Le développement durable, de l'utopie au concept. De nouveaux chantiers pour la recherche », extrait du colloque organisé par Natures Sciences Sociétés en 1997, Natures-SciencesSociétés, Paris, Elsevier, p. 131-142.

Wackermann G., 2002, Géographie régionale, Paris, Ellipses, coll. U Géographie, 240 p.

Wackermann G., 1989, Le Pays de la potasse. Des lieux et des hommes, Illkirch, Le Verger éditeur, 112 p.

Zaccaï E., 2004, « Les indicateurs de développement durable » in Le dictionnaire du développement durable, Institut pour un Développement Durable- Ottignie/ Centrum voor Duurzaame Ontwickeling - RUG. SSTC, www.billy-globe.org/fr_2001/ dico-dd_I.D.D. -final-def\%20doc.pdf, 2003, 8 p.

Zuindeau B., 2000, Développement durable et territoire, Lille, Presses Universitaires Septentrion, 289 p.

Zuindeau B., 1997, «Le développement durable : les enseignements de l'approche spatiale », Communication pour le $45^{\text {ème }}$ séminaire de l'OIPR, Lille, Centre Economie et politiques européennes, Université des Sciences et Technologies, 16 p.

\section{RÉSUMÉS}

Malgré les nombreux soutiens méthodologiques à l'élaboration de l'Agenda 21 local, bon nombre de collectivités territoriales ont toujours des difficultés à choisir une démarche optimale de mise en œuvre $\mathrm{du}$ développement durable. Quelle échelle territoriale, quels objectifs de développement durable, quelle évaluation adopter ? Pour répondre à ces questions, cet article présente les étapes essentielles d'un diagnostic dit de développement durable adaptable à tout type de territoire. La théorie a ensuite été confrontée aux réalités du terrain à partir d'un 
exemple de territoire de reconversion considéré comme pertinent pour la mise en œuvre d'un plan de développement durable: le Bassin potassique alsacien.

Although several methodological helps for the local Agenda 21, many local governments have still difficulties to plan the sustainable development for their territory. Which territorial scale, which sustainable development aims, which evaluation to apply ? To reply, this article introduces the essential stages of a sustainable development diagnosis which can adjust to any territory. The theory has been confronted to the ground reality thanks to an example of regeneration territory considered as pertinent for a sustainable development plan: the Alsace potassium Basin.

\section{INDEX}

Mots-clés : territoire, diagnostic territorial, indicateurs de développement durable, bassin potassique alsacien

Keywords : territory, territorial diagnosis, sustainable development indicators, Alsace potassium Basin

\section{AUTEUR}

\section{FRANÇOIS BESANCENOT}

François Besancenot est docteur en géographie de 1,ENS-Lettres et Sciences humaines (Lyon). Il mène actuellement des recherches sur le territoire durable et l,éducation au développement durable au sein de l,UMR 8185 Espaces, Nature et Culture (CNRS et Université Paris IV-Sorbonne). Il est aussi producteur d,émissions dédiées aux bonnes pratiques pour la société Nature Prod. Courriel : francois.besancenot@yahoo.fr 